Relations industrielles

Industrial Relations

\title{
Le Mouvement syndical en Belgique, par Guy Spitaels, deuxième tirage, Bruxelles, Éditions de l'Institut de Sociologie, Université libre de Bruxelles, 1969, 96 pp.
}

\section{Gérard Dion}

Volume 25, numéro 3, 1970

URI : https://id.erudit.org/iderudit/028161ar

DOI : https://doi.org/10.7202/028161ar

Aller au sommaire du numéro

Éditeur(s)

Département des relations industrielles de l'Université Laval

ISSN

0034-379X (imprimé)

1703-8138 (numérique)

Découvrir la revue

Citer ce compte rendu

Dion, G. (1970). Compte rendu de [Le Mouvement syndical en Belgique, par Guy Spitaels, deuxième tirage, Bruxelles, Éditions de l'Institut de Sociologie,

Université libre de Bruxelles, 1969, 96 pp.] Relations industrielles / Industrial Relations, 25(3), 621-621. https://doi.org/10.7202/028161ar

Tous droits réservés @ Département des relations industrielles de l'Université Laval, 1970
Ce document est protégé par la loi sur le droit d'auteur. L'utilisation des services d'Érudit (y compris la reproduction) est assujettie à sa politique d'utilisation que vous pouvez consulter en ligne.

https://apropos.erudit.org/fr/usagers/politique-dutilisation/ 


\section{RECENSIONS}

Le Mouvement syndical en Belgique, par Guy Spitaels, deuxième tirage. Bruxelles, Editions de l'Institut de Sociologie, Université libre de Bruxelles, $1969,96 \mathrm{pp}$.

Cette étude, visiblement préparée pour des lecteurs étrangers à la Belgique, est d'un intérêt particulier pour ceux qui veulent connaître les données essentielles du mouvement syndical belge.

L'auteur présente d'abord l'histoire des origines du syndicalisme jusqu'au lendemain de la guerre 1939-1945. Ensuite, selon un schéma identique qui permet facilement des comparaisons (organisation statutaire, effectifs, objectifs économiques et sociaux, liens politiques), il aborde les trois centrales ouvrières : Fédération générale du travail de Belgique, Confédération des syndicats chrétiens, Centrale générale des syndicats libéraux de Belgique. Enfin, il dresse un bilan de l'acquis et des défis du syndicalisme en ce pays.

L'ouvrage est bien charpenté et l'auteur, tout en étant objectif, ne craint pas de porter des jugements de valeur à partir de sa propre conception du syndicalisme, conception que nous partageons. Une question de détail : nous nous expliquons difficilement pourquoi il fait remonter la création de la CSC en 1912 alors que, selon le critère utilisé pour la CGTB (p. 13), il nous apparaît que cela devrait être en 1904 (p. 18).

Tous ceux qu'intéresse le développement du mouvement syndical ont avantage à connaître l'expérience belge.

Gérard DION

Relations humaines et relations industrielles, par Marcel Bolle $\mathrm{De} \mathrm{Bal,}$ $2 \mathrm{e}$ édition, Bruxelles, Etudes de sociologie du travail, Editions de l'Institut de Sociologie, Université Libre de Bruxelles, 1969, 146 pp.

De cet essai, Marcel Bolle de Bal n'a rien voulu modifier pour cette réédition dont la première publication remonte à
1958, car ses réflexions témoignent d'un moment de l'évolution des idées de la sociologie du travail. L'auteur constate que les «relations humaines》 et la sociologie du travail ont tendance à être confondues en une seule et même chose ; il s'efforce de mettre en évidence les causes de cette assimilation.

Sa première démarche consiste en un essai de définition théorique des divers aspects de 《relations humaines》. Ces aspects sont, en fait, cinq notions différentes que recouvre cette discipline: l'existence, l'analyse méthodique, les techniques, la politique et l'esprit des « relations humaines 》. Cette définition théorique est présentée à la lumière d'un rappel historique du mouvement, lequel doit sa naissance et son essor à des motifs presque uniquement économiques ; cela explique, en partie, les déficiences du côté de l'analyse du cadre théorique, les 《relations humaines 》 n'ayant été développées que dans une optique pratique.

L'auteur expose également le problème des rapports entre « relations humaines $\gg$ - entendues au sens des interrelations psychologiques et sociales des individus ou des groupes au travail, et « relations industrielles 》, - relations entre organisations professionnelles d'employeurs et de travailleurs - . Le problème se noue autour du désaccord qui apparaît entre syndicats et employeurs à propos des motifs « humanitaristes ou humanistes », qui inspirent les politiques de relations humaines. On assiste alors à des prises de positions contradictoires vis-à-vis des relations industrielles, les syndicats considérant ces dernières comme une condition nécessaire aux «bonnes relations humaines 》.

Face à cette opposition qui semble irréductible, l'auteur propose une solution constructive. Ces conflits de valeurs canalisés et orientés, deviennent générateurs de progrès social, dont les deux parties, à la fois, peuvent tirer profit.

Ses réflexions amènent l'auteur à conclure que les « relations humaines », bien que nécessaires, ne suffisent pas à solu- 